\title{
A KNOWLEDGE-BASED APPROACH ON GLOBELAND30 INCREMENTAL UPDATING: A CASE STUDY OF BUILT-UP AREA
}

\author{
Jun Zhang *, Hao Wu, Shu Peng \\ National Geomatics Center of China, Beijing, China - (junzhang, wuhao, pengshu)@nsdi.gov.cn
}

Theme Sessions, ThS 18

KEY WORDS: Globeland30, incremental updating, Built-up area, Segmentation, Domain knowledge

\begin{abstract}
:
Global land cover/land use product in multiple periods is pivotal to understand the complex drivers and mechanisms in global climate change, and to forecast future land use trends in sustainable development. GlobeLand30, as the world's first high spatial resolution land cover product ( $83 \%$ accuracy), needs to be continually updated to meet various needs. However, many challenges such as removing pseudo change to keep consistency of updating - remain unsolved. To deal with high temporal and spatial variability happened within built-up area class and between it and other classes, this paper presents an alternative approach that exploits domain knowledge and object-based change detection technique. The central premise of the approach is that one-class segmentation is first proceeded on both former image and current image. Then, segments of former image are labeled by using corresponding Globeland30 product. Segments of built-up area in current image are finally recognized through correlation which is established based on domain knowledge. Knowledge used in this study mainly includes area index, shape index, perimeter index, spectral similarity, 'from to' types and spatial relation. The proposed method and classification method were tested for their ability for built-up area updating in Shandong area. Results showed that the proposed method proved particularly effective for maintaining consistency of unchanged areas from former product to current one, and more than $80 \%$ changes could be identified correctly. The proposed method also provided a practical way for an economic and accurate updating of Globeland30 product.
\end{abstract}

\section{INTRODUCTION}

Global land cover/land use product in multiple periods (i.e. three or more) is pivotal to understand the complex drivers and mechanisms in global climate change, and to forecast future land use trends in sustainable development (Chameides et al., 1994; Bianchin et al., 2008). Remote sensing image with different spatial resolutions has been used in land cover/land use mapping in recent decades. Taking into account of availability, coverage and rich spatial details of requirements of remotely sensed images, $1 \mathrm{~km}$ to $30 \mathrm{~m}$ spatial resolutions are usually chosen for global land cover/land use mapping and change detection (Small, 2005).

In recent years, multiple sets of global land cover product have been produced, from 1 kilometres to $30 \mathrm{~m}$ spatial resolution(Hansen et al., 2000; Friedl et al., 2010; Bicheron et al., 2012). In 2015, a POK-based operational approach has been proposed to create a global land cover map, the GlobeLand30, the world's first high spatial resolution $(30 \mathrm{~m})$ land cover product with 2000 and 2010 two periods. Compared with former products, GlobeLand30 is one order of magnitude higher in spatial resolution. The overall classification accuracy reached more than $80 \%$. GlobeLand 30 provides a solid research foundation for geography monitoring and other applications (Chen et al., 2015).

GlobeLand30 needs to be continually and timely updated to meet various needs. However, 1) many challenges - such as removing pseudo change to keep temporal-spatial consistency of updating - remain unsolved; 2) most of researchers mainly focused on changed area detection for updating(Singh et al., 1988; Carlotto 1997), while detecting unchanged area in new image is equal important for changed area detection is always with some errors. In details, pseudo change generally happens in unchanged area and caused by temporal different between former image and new image. For example, harvested farmland type and built-up area type have similar spectral feature, pseudo change may happened in the area where is harvested farmland cover with result of being recognized as built-up area. Temporal consistency here means that the updated products should be the same as the products of subsequent base line years in unchanged areas (Wang et al., 2014).

Usually, there are two kinds of methods for removing pseudo change. First, relative radiometric normalization has been proceeded between bi-temporal images to reduce temporal differences (Canty et al., 2008). Second, spectral-temporal feature has been extracted from time-series images in order to increase separability of inter-classes for reducing probability of pseudo change (Kennedy et al., 2010). There are advantages of these methods for removing pseudo change to a certain extent. However, it is a time and labour-consuming work to construct time-series feature for land cover mapping in the global extent. In addition, there are problems in methods for detecting changed area. Hussain (2013) make a review of methods including three aspects, pixel based, objects-based and data mining. Objects-based change detection technique has been often adopted for advantage of keep integrity of meaningful regions (segments).

\footnotetext{
* Corresponding author
} 
During last two decades, knowledge has been investigated to improve the quality of land cover mapping. In general, there are two kinds of knowledge, one is image-based knowledge, such as spatial relations between different land cover types or image objects, and another is geospatial knowledge, such as associated rules summarized with ancillary data (Chen, 1984; Harris and Ventura, 1995; Lu and Weng, 2007; Kantakumar et al., 2016, zww). In the present article, we focused on the image-based knowledge. However, most of studies focus on methods of measuring spatial relationship (Groom et al., 1994; Qiao and Wang, 2014). The knowledge summarization is not insufficient for global land cover updating, and even more severe in finer resolution $(30-\mathrm{m})$.

Therefore, in the present paper, object-based change detection strategy is first adopted, domain knowledge structure for global land cover updating has been exploited, knowledge rules has been summarized and used in updating procedure, the unchanged area and changed area are separately detected, pseudo change are reduced, and finally an alternative approach for GlobeLand30 updating is proposed.

\section{METHODOLOGY}

The central premise of the approach is that one-class segmentation is first proceeded on both former image and current image. Then, segments of former image are labeled by using corresponding Globeland30 product. Segments of builtup area in current image are finally recognized through correlation between bi-temporal segments, the correlation is established based on domain knowledge of built-up area class. The proposed method relies on a segmentation algorithm that provides high precise results for each temporal image, and relies on domain knowledge which supports to identify both unchanged segments and changed types from former image to current image. Procedure used in this study is shown in Figure 1.

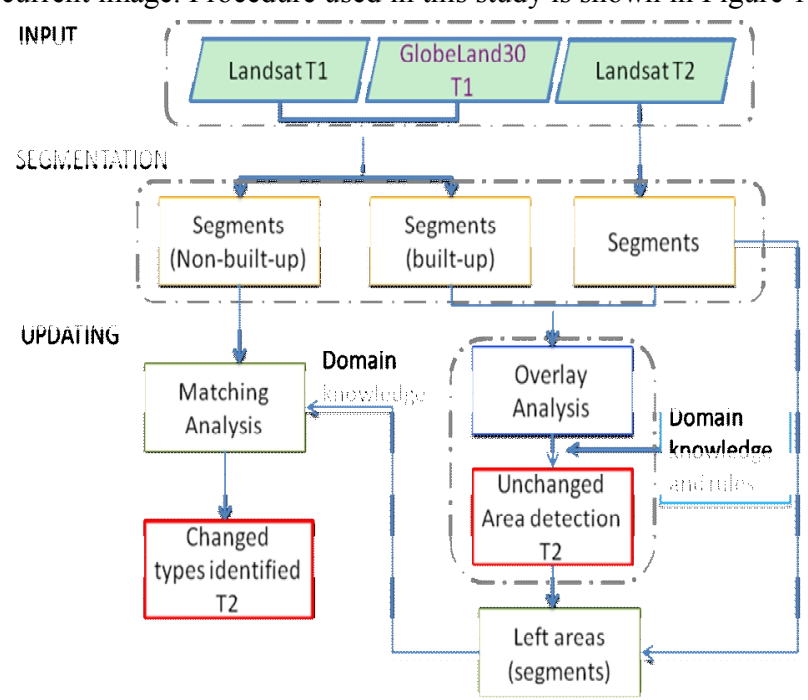

Figure 1. Globeland30 updating procedure in this study

\subsection{Image Segmentation}

A prerequisite step of the proposed method is image segmentation, which partitions an image into a number of homogeneous and meaningful regions (segments) (Li and Xiao, 2007). Segmentation is first proceeded on both former image and current image. Segmentation parameters has been selected and threshold value is adjusted for one certain land cover type, e.g. built-up area class. Segments of former image are labeled as two classes: built-up area class and non-built-up area class, through overly analysis with corresponding Globeland30 product. Segments of current image are unlabeled in this step.

Two segmentation methods are used for each image: multiresolution segmentation from eCognition, a hierarchical image segmentation (Rahman et al., 2008; Li et al., 2011). Multiresolution segmentation is a bottom-up segmentation based on a pairwise region merging technique. It minimizes the average heterogeneity and maximizes their respective homogeneity, through adjusting value of four parameters: scale, image layer weights, shape and compactness. A hierarchical image segmentation is a multi-scale and multilevel segmentation, which combines multichannel watershed transformation ( $\mathrm{Li}$ and Xiao, 2007) and dynamics of watershed contours (Najman and Schmitt, 1996). However, other image segmentation methods can also be used to produce results.

In present research, after the comparison of the segments from the two segmentation methods, the one with better boundary matching for built-up class would be selected and used in subsequent processing.

\subsection{Domain Knowledge}

In order to make a continuous and timely land cover updating in the global extent, a set of domain knowledge is expected to support in image acquisition, image processing, land cover updating, validation and feature description in different regions / economical zones / countries. Domain knowledge presented in the paper indicates a knowledge system for the global land cover updating research field, which specially relates to the knowledge based on image, consisting of several knowledge bases, such as image knowledge base, sample knowledge base, method knowledge base, land cover (LC) knowledge base and relations knowledge base (Figure 2).

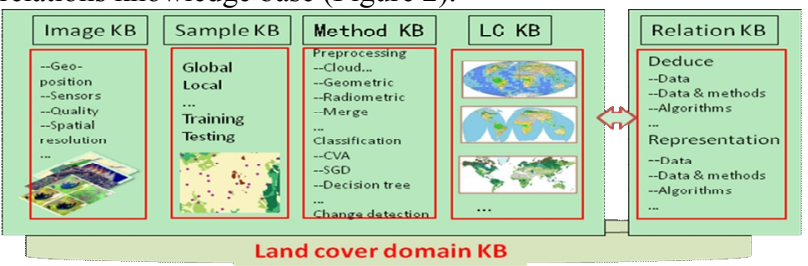

Figure 2. Major components of domain knowledge

Relations knowledge is mainly consisted of three components, 1) relations between objects/land cover types in the image, 2) relations between image and method, 3) relations between methods/algorithms. In the present paper, the first one was adopted, knowledge of built-up area including area index, shape index, perimeter index, spectral similarity, 'from to' relation and spatial relation, had been used in this study.

\subsection{Updating with Knowledge Rules}

In the updating procedure, the unchanged area and changed area were detected and recognized with Knowledge rules. The unchanged area detection was processed mainly by overly analysis between built-up area segments in T1 and segments in $\mathrm{T} 2$, through usage of area index, shape index and perimeter index. One assumption before using knowledge rules is that the built-up area is the land cover type which won't degradation in following several years (e.g. ten years). Knowledge rules used in this step contains: 1) two compared segments should meet: 
AreaT2-AreaT1 $_{\mathrm{T}}=0$; 2) Shape $_{\mathrm{T} 2}$ is more or less equal to Shape $\mathrm{T}_{2}$, which shows built-up area segment $\mathrm{T}_{1}$ enlarged in $\mathrm{T} 2$ or keep the same; 3) Perimeter $\mathrm{T} 2-$ PerimeterT1 $>=0$. If the three rules are satisfied, segmentsт2 which is the same as or larger compared with built-up area segment $\mathrm{T}_{\mathrm{1}}$ will be labeled as built-up area. The overly percent is calculated by area comparison.

The changed area, as the new built-up area in T2, is detected from the rest of segments from last step. In this step, knowledge of spectral similarity, 'from to' relation and spatial relation were used. Knowledge rules can be summarized as: 1) segmentsт2 should has different spectral feature compared with non-built up segments 11 , spectral similarity is measured by Euclidean distance function with bi-temporal spectral bands; 2) 'from to' relation, such as large area of water is almost impossible changed to built-up area, 3) space distance of segmentsT2 from the edge of city or the nearest built-up area, which is higher than a threshold value $\mathrm{h}$, is rarely be built-up area.

\subsection{Evaluation}

In order to evaluate the proposed method, the proposed method and classification method were tested for their ability to update

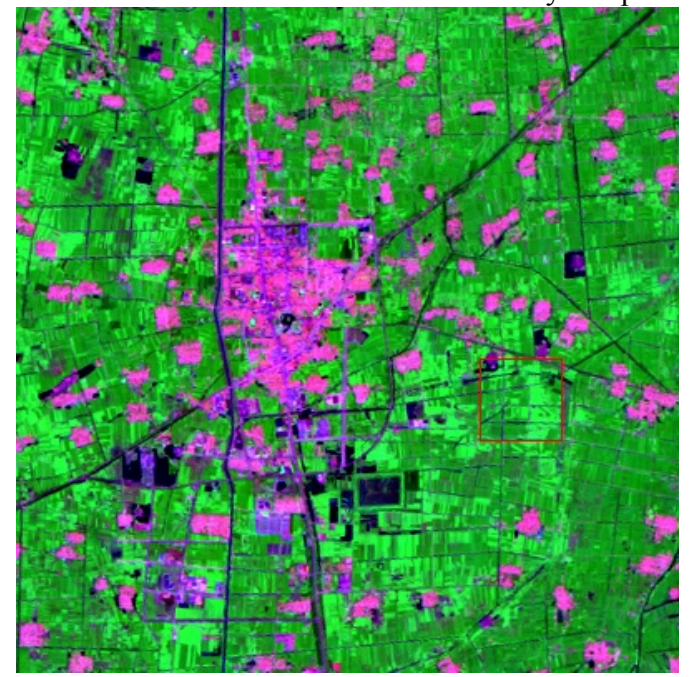

built-up area. Classifiers of the maximum likelihood (ML) method was used in this study. Since the ML method is a classical classifier and have been widely used in many applications (Guindon and Gascuel, 2003). A set of testing samples was used as reference data to calculate the overall accuracy.

\section{STUDY AREA AND DATA}

The case study was processed in Shandong province, in the north of China. The mainly land cover types in this area are farmland, built-up area, water and grassland.

In this study, Landsat OLI data acquired on 2010 and 2013 were selected as experimental data. The OLI data include six multispectral bands with spatial resolution of 30 meters. An image subset of $600 \times 610$ pixels was finally used. Figure 3 shows a false-colour composite, illustrating urban landscapes. The GlobeLand30 -2010 product in this area is also acquired as input.

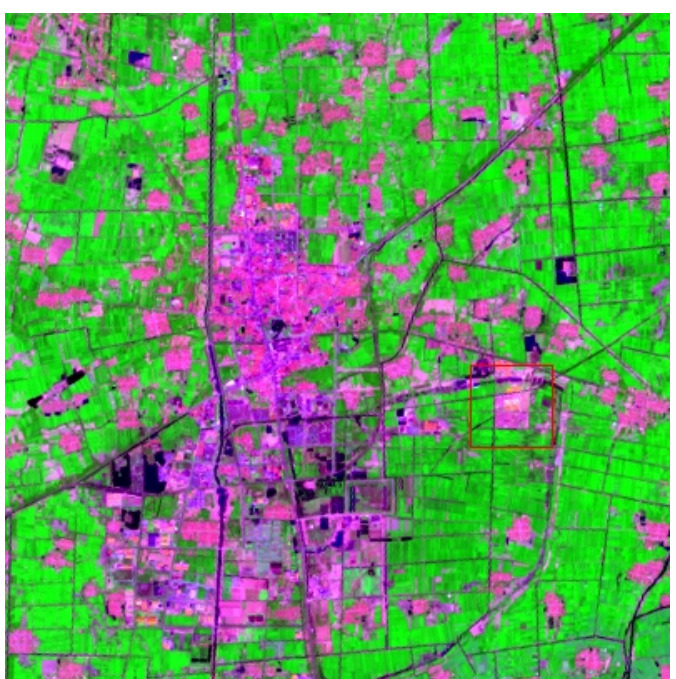

Figure 3. Landsat OLI image of Shandong urban area (band 6, 4 and 2 as R, G and B); left: acquisition in 2010 and right 2013

\section{EXPERIMENTAL VALIDATION}

\subsection{Image Segmentation}

As mentioned before, when processing image segmentation using multi-resolution segmentation method, several parameters need to be considered: scale, image layer weights, shape and compactness, as well as scale parameter for hierarchical segmentation method.

In this paper in order to choose an appropriate value of parameters for final image segmentation and subsequent updating, several sets of value were respectively used in image segmentation and compared in terms of visual interpretation. It was found that the multi-resolution segmentation method with scale parameter of 200, image layer weights of 1 , shape of 0.1 and compactness of 0.5 achieved the relatively better boundary matching for the certain land cover types, built-up area.

\subsection{Evaluation of Built-up Area Updating}

Two built-up area updating results were obtained from the proposed method and one classification method. A set of 18,354 pixels as testing sample was used in overall accuracy calculating. As a result, $73 \%$ of built-up area was updated with classification method in 2013 , while more than $80 \%$ of built-up area was updated with the proposed method.

Figure 4(a), (b) and (c) show GlobeLand30 product of 2010 in Shandong, classification results of 2013 by ML method, builtup area updating results in 2013 by the proposed method respectively. Figure 4(d) shows the Landsat OLI image of Shandong urban area in 2013, the two highlighted rectangle areas are mostly of changed built-up area. In the Figure 4(b), only one of the changed built-up area (right) can be extracted, the left one almost cannot be detected correctly by the classification method. In the Figure 4(c), both of updated builtup areas were recognized more correctly by the proposed method. This result also validates the performance of the proposed method. 


\section{CONCLUSION}

This paper proposed an alternative approach that exploits domain knowledge and rules, as well as object-based change detection technique for Globeland30 updating. Results showed that the proposed method proved particularly effective for maintaining consistency of unchanged areas from former product to current one, and more than $80 \%$ built-up area changes could be identified correctly. The proposed method also provided a practical way for an economic and accurate updating of Globeland30 product. In future work, the knowledge base should be continuously developed, the automatic technique of threshold determination in knowledge rules should attract more attention.

\section{REFERENCES}

Bianchin, A., \& Bravin, L. (2008). Remote sensing and urban analysis. Lecture Notes in Computer Science, 5072, 300-315.

Bicheron P, Defourny P, Brockmann C, Vancutsem C, Huc M, Bontemps S, Leroy M, Achard F, Herrold M, Ranera F, Arino O. (2008). GLOBCOVER: Products description and validation report. Toulouse, France MEDIAS-France.

Carlotto, M. J. (1997). Detection and analysis of change in remotely sensed imagery with application to wide area surveillance.. IEEE Transactions on Image Processing, 6(6), 189-202.

Canty, M. J., \& Nielsen, A. A. (2008). Automatic radiometric normalization of multi-temporal satellite imagery with the iteratively re-weighted mad transformation. Remote Sensing of Environment, 112(3), 1025-1036.

Chameides, W.L., Kasibhatla P., Yienger J., et al.(1994). Growth of continental-scale metro-agro-plexes, regional ozone pollution, and world food production. Science, 264(5155): 7477.

Chen, J. (1984). The application of DTM in improving classification accuracy of remote sensing imagery. Geomatics \& Information Science of Wuhan University.

Chen, J, Chen J, Liao A, et al. (2015). Global land cover mapping at $30 \mathrm{~m}$ resolution: A POK-based operational approach. ISPRS Journal of Photogrammetry \& Remote Sensing, 103:7-27.

D. Lu, \& Q. Weng. (2007). A survey of image classification methods and techniques for improving classification performance. International Journal of Remote Sensing, 28(5), 823-870.

Friedl, M. A., Sulla-Menashe, D., Tan, B., Schneider, A., Ramankutty, N., Sibley, A., et al. (2010). MODIS collection 5 global land cover: algorithm refinements and characterization of new datasets. Remote Sensing of Environment, 114(1), 168-182.

Groom, G. B., Fuller, R. M., Jones, A. R. (1996) Contextual correction: techniques for improving land cover mapping from remotely sensed images. International Journal of Remote Sensing, 17(1), 69-89.

Guindon, S., \& Gascuel, O. (2003). A simple, fast, and accurate algorithm to estimate large phylogenies by maximum likelihood.. Systematic Biology, 52(5), 696-704.
Li, P., \& Xiao, X. (2007). Multispectral image segmentation by a multichannel watershed-based approach. International Journal of Remote Sensing, 28(19), 4429-4452(24).

Li, P., Guo, J., Song, B., \& Xiao, X. (2011). A multilevel hierarchical image segmentation method for urban impervious surface mapping using very high resolution imagery. IEEE Journal of Selected Topics in Applied Earth Observations \& Remote Sensing, 4(1), 103-116.

Lu, D. \& Weng, Q. (2007). A survey of image classification methods and techniques for improving classification performance. International Journal of Remote Sensing, 28(5), 823-870.

Hansen, M. C., Defries $\dagger$, R. S., And, J. R. G. T., Sohlberg, R. (2000). Global land cover classification at $1 \mathrm{KM}$ spatial resolution using a classification tree approach. International Journal of Remote Sensing, 21(6), 1331-1364.

Harris, P. M., \&Ventura, S. J. (1995). The integration of geographic data with remotely sensed imagery to improve classification in an urban area. Photogrammetric Engineering and Remote Sensing, 61: 993-998.

Hussain, M., Chen, D., Cheng, A., Wei, H., \& Stanley, D. (2013). Change detection from remotely sensed images: from pixel-based to object-based approaches. ISPRS Journal of Photogrammetry \& Remote Sensing, 80(2), 91-106.

Kantakumar, L. N., Kumar, S., \& Schneider, K. (2016). Spatiotemporal urban expansion in pune metropolis, India using remote sensing. Habitat International, 51, 11-22.

Kennedy, R. E., Yang, Z., \& Cohen, W. B. (2010). Detecting trends in forest disturbance and recovery using yearly Landsat time series: 1. Landtrendr- temporal segmentation algorithms. Remote Sensing of Environment, 114(12), 2897-2910.

Najman, L., \& Schmitt, M. (1996). Geodesic saliency of watershed contours and hierarchical segmentation. IEEE Transactions on Pattern Analysis \& Machine Intelligence, 18(12), 1163-1173.

Qiao, C., \& Wang, J. (2014). Spatial relationship-assisted classification from high-resolution remote sensing imagery. Infection \& Immunity, 55(5), 1-17.

Rahman, M. R., \& Saha, S. K. (2008). Multi-resolution segmentation for object-based classification and accuracy assessment of land use/land cover classification using remotely sensed data. Journal of the Indian Society of Remote Sensing, 36(2), 189-201.

Singh, A., \& Singh, A. (1988). Digital change detection techniques using remotely sensed data. International Journal of Remote Sensing, 10(6), 989-1003.

Small, C. (2005). A global analysis of urban reflectance. International Journal of Remote Sensing, 26(4), 661-681.

Wang, J., Zhao, Y., Li, C., Yu, L., Liu, D., \& Gong, P. (2014). Mapping global land cover in 2001 and 2010 with spatialtemporal consistency at $250 \mathrm{~m}$ resolution. ISPRS Journal of Photogrammetry \& Remote Sensing, 103(4-8), 38-47. 

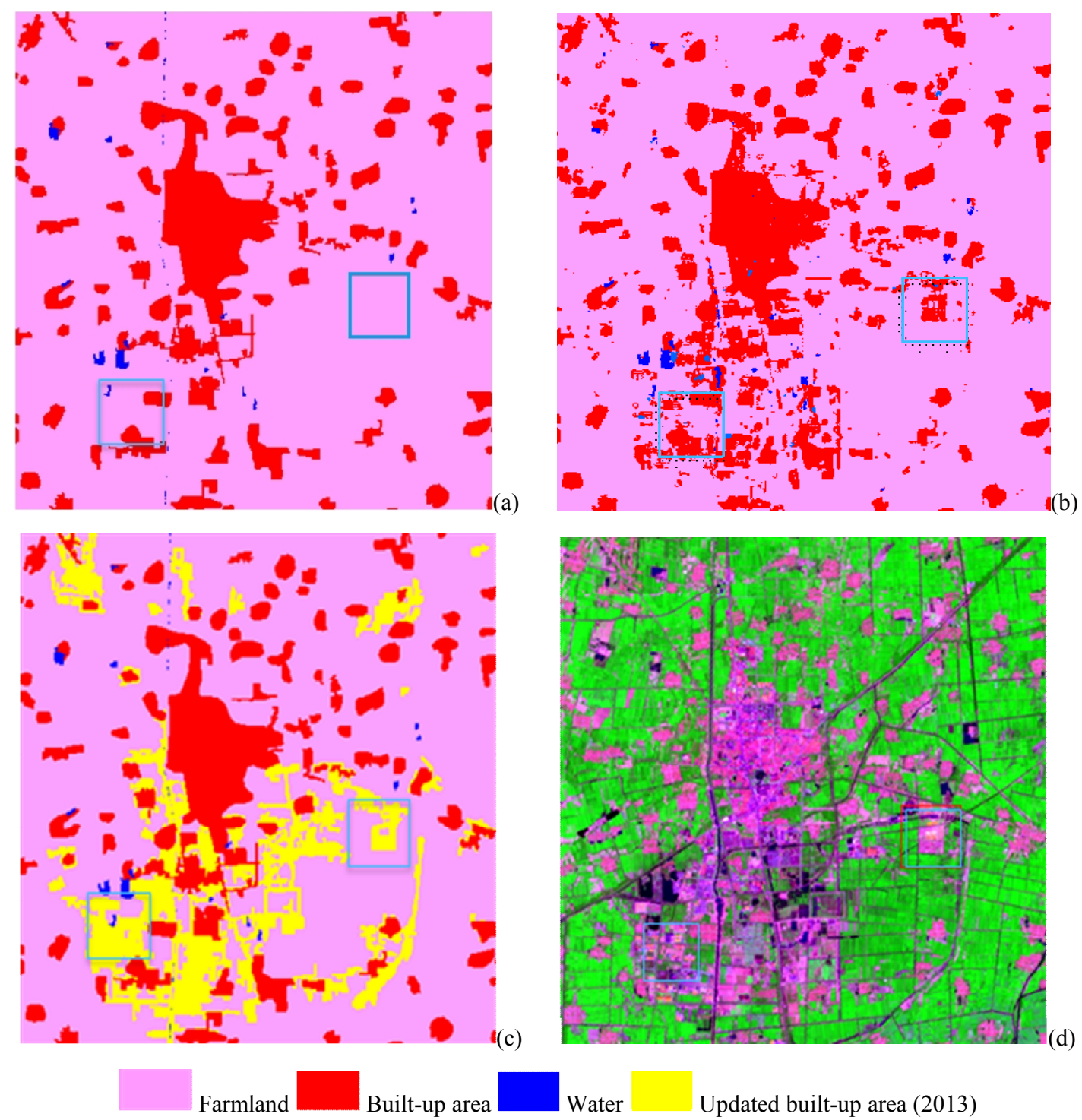

Figure 4. GlobeLand30 product of 2010 in Shandong (a), classification results of 2013 by ML method (b), built-up area updating results in 2013 by the proposed method (c), the Landsat OLI image of Shandong urban area in 2013(d). 